\title{
Retrospective Monitoring in the Management of Persistent Asthma
}

\author{
Georgios Kourtis MD, Maria-Filomena Caiaffa MD PhD, Cesarea Forte RN, \\ Marisa I Scarlato MD, and Luigi Macchia MD PhD
}

\begin{abstract}
BACKGROUND: There is still considerable disagreement on several aspects of monitoring asthma with symptom score and peak expiratory flow (PEF). OBJECTIVE: To investigate patient adherence to a retrospective diary-card monitoring method in patients with poorly controlled persistent asthma, in a clinical management setting; to develop improved methods for fast manual entry data into a computer; and to generate real-time informative graphs of the data. METHODS: In 115 consecutive adult patients we applied a diary-card monitoring method in which the patient records symptom score and PEF. We analyzed the diary cards of 84 patients. We used SigmaPlot software to graph the data, and linear regression to analyze the relationship between days of expected diary-card completion and days of actual correct diary-card completion (completed entries). RESULTS: Linear regression gave an overall correlation coefficient $\left(r^{2}\right)$ of 0.65 . Surprisingly, the $\mathbf{r}^{2}$ values in the patients with mild, moderate, and severe asthma were $0.24,0.44$, and 0.99 , respectively, revealing a striking correlation between adherence and severity. Moreover, when we arbitrarily set $75 \%$ as the minimum acceptable rate of days of completed diary-card entries, $68 \%$ of the patients were in the over-75\% category. Remarkably, $100 \%$ of the patients with severe asthma were above the $75 \%$ cut-off. The graphing method we tested proved user-friendly, flexible, and quick, allowing computerized processing of 30 days of data sets in $5 \mathrm{~min}$, and generation of high-quality selfexplanatory graphs that facilitate rapid management decision making via visual pattern recognition. CONCLUSIONS: In a clinical setting, retrospective monitoring of patients with moderate and severe persistent asthma by symptom score and PEF is feasible, and patient adherence appears to be good, particularly in patients with severe asthma. We recommend a lower priority on retrospective monitoring in patients with mild persistent asthma. Monitoring should be carried out according to a definite follow-up protocol. Improving the quality and standardization of the monitoring graph is a priority. Key words: severe asthma; symptom score; PEF; monitoring; adherence; diary. [Respir Care 2011;56(5):633-643. (C) 2011 Daedalus Enterprises]
\end{abstract}

\section{Introduction}

Asthma is a chronic inflammatory disorder of the airways, characterized by bronchial hyper-responsiveness, airflow limitation, and respiratory symptoms. Persistent in-

Georgios Kourtis MD, Maria-Filomena Caiaffa MD PhD, Cesarea Forte $\mathrm{RN}$, Marisa I Scarlato MD, and Luigi Macchia MD $\mathrm{PhD}$ are affiliated with the Department of Allergology and Clinical Immunology, University of Bari, Bari, Italy. Dr Caiaffa is also affiliated with the Department of Allergology and Clinical Immunology, University of Foggia, Foggia, Italy.

Dr Kourtis presented a version of this paper at the 26th Congress of the European Academy of Allergology and Clinical Immunology, held June 9-13, 2007, in Göteborg, Sweden. flammation produces respiratory impairment through repeated (albeit reversible) acute bronchoconstriction and airway remodeling. These and other pathogenetic mechanisms have important consequences on asthma prevention, diagnosis, therapy, and management. Exacerbations occur with variable frequency, and long-term treatment and man-

\footnotetext{
Drs Kourtis, Caiaffa, and Macchia have disclosed relationships with Novartis. The other authors have disclosed no conflicts of interest.

Correspondence: Luigi Macchia MD PhD, Department of Allergology and Clinical Immunology, Policlinico, 70124 Bari, Italy. E-mail: 1.macchia@allergy.uniba.it.
}

DOI: $10.4187 /$ respcare. 00796 
University of Bari

MIDIM Department. Allergology and Clinical Immunology Section.

Clinical Unit of Allergology and Clinical Immunology

Head: Prof. Angelo Vacca

Head: Prof. Luigi Macch Treatment Center

Policlinico, Piazza Giulio Cesare

70124 Bari

Name

Year:

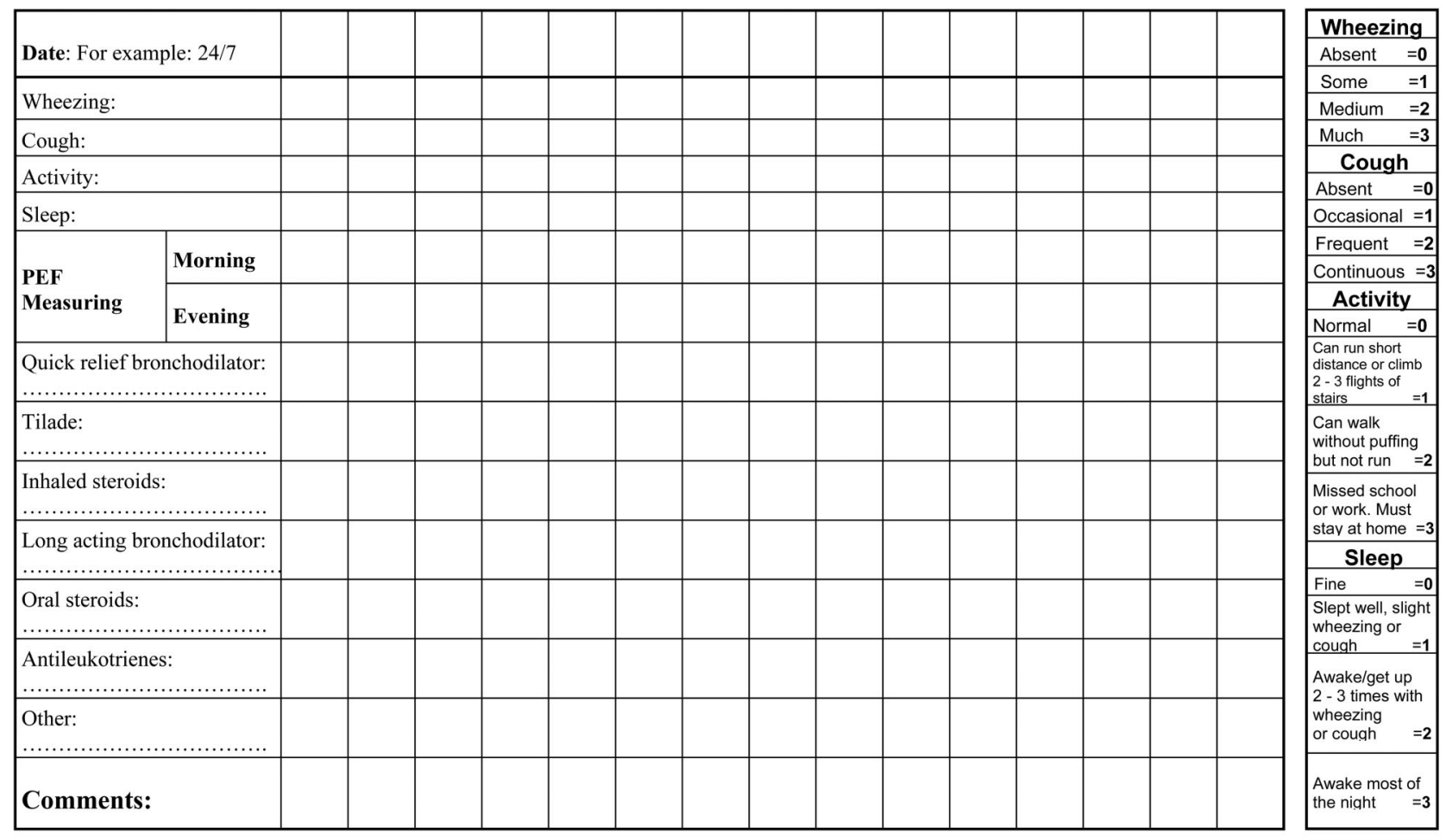

Fig. 1. Asthma monitoring diary card used in our asthma clinics. (Adapted from Reference 1.)

agement are necessary to stabilize symptoms and improve function, ${ }^{1-3}$ so asthma requires appropriate diagnosis and treatment at initial presentation, and accurate long-term follow-up with regular control visits.

Various prospective and retrospective asthma monitoring methods, usually based on recording of symptom score and peak expiratory flow (PEF) measurements, have been proposed, and their implementation in the clinical practice is strongly recommended, particularly in patients with worse than mild asthma. ${ }^{1-4}$ Prospective monitoring, in association with an asthma action plan, is one of the most important self-management methods and improves outcomes and quality of life of asthmatic patients. . $^{5-7}$

On the other hand, retrospective monitoring allows fine diagnosis assessment and punctual therapy evaluation. ${ }^{8-11}$ However, its application to routine clinical practice poses several still controversial questions: particularly, to whom and exactly when should retrospective monitoring be applied? For how long? How should the information returned by patients at follow-up visits be processed (eg, graphing, statistical analysis)? And how can the processed information be made immediately available to the medical personnel in charge of management? Moreover, there is little consensus on the standardization of graphs obtained through recording of symptom score and PEF. ${ }^{11}$

We applied a retrospective asthma-monitoring method, based on daily patient recording of symptom score and PEF on a diary card, in all new patients with poorly controlled persistent asthma, according to the Global Initiative for Asthma (GINA) ${ }^{4}$ definition. These patients were consecutively seen during a 13-month period at our two asthma clinics, at the University of Bari, Bari, Italy, and the University of Foggia, Foggia, Italy. We developed a modified version of the diary card proposed in 1997 in the United States National Institutes of Health Guidelines for the Diagnosis and Management of Asthma (Fig. 1). ${ }^{1}$ Patients were asked to daily record symptom score and PEF for a period of time according to their asthma severity. At follow-up visits, we immediately processed the diary-card data and generated one or more monthly graphs of the patient's status and performance for that diary-card period. Here we present and discuss the method we developed and analyze patient adherence to this method. We also surveyed the patients on their opinions about the diary-card 


\section{Retrospective Monitoring in the Management of Persistent Asthma}

monitoring method and its impact on their quality of life, via mail questionnaire, answered anonymously.

\section{Methods}

This study was approved by the ethics committee of the University of Foggia. Though the data were collected within our usual asthma monitoring protocol, in this study all patients gave formal informed consent, in observance of the Declaration of Helsinki.

This was an observational study in which we analyzed some of the outcomes of a home asthma monitoring method that is based on the patient's daily filling in their symptom score and morning and evening PEF on a diary card. We studied 84 consecutive adult patients with poorly controlled persistent asthma. The patients were seen at either the Foggia or Bari asthma clinic, depending on their domicile. We have routinely used this kind of asthma monitoring method at both centers since their establishment (Bari 1986, Foggia 2001). As part of our permanent collaboration, between November 2004 and February 2005, the staff teams:

- Developed an improved diary card

- Improved the method for immediate analysis and graphing of diary-card data at follow-up visits

- Defined more precise application criteria for the monitoring method (eg, exclude patients with intermittent asthma or purely seasonal asthma, and routinely apply the method with all patients with persistent asthma)

- Defined the protocol's asthma-monitoring duration, according to the patient's asthma severity

We implemented the revised method in both clinics in March 2005, and after 13 months we assessed the revised method, particularly patient adherence to the diary-card method, patients' perceptions about the method, and our data gathering, analysis, and graphing methods.

\section{Clinical Work}

The clinical work was carried out by 4 physicians with comprehensive experience in asthma treatment, and a student nurse. The defining criterion for asthma diagnosis was recurrent and clinically manifest bronchoconstriction that was reversible spontaneously or with treatment. Asthma was classified as intermittent, mild persistent, moderate persistent, or severe persistent, based on clinical and functional indexes of asthma severity over the preceding year, according to the GINA 2002 guidelines. ${ }^{4}$ Seasonal asthma was defined as asthma that occurred only intermittently, often in association with allergic rhinitis, with the patient being asymptomatic in other seasons. ${ }^{4}$
Table 1. New Patients With Suspected Asthma: March 2005 to March 2006

\begin{tabular}{lccc}
\hline \hline \multicolumn{1}{c}{ Diagnosis } & $\begin{array}{c}\text { Bari } \\
\text { Clinic } \\
\text { no. }(\%)\end{array}$ & $\begin{array}{c}\text { Foggia } \\
\text { Clinic } \\
\text { no. }(\%)\end{array}$ & $\begin{array}{c}\text { Total } \\
\text { no. }(\%)\end{array}$ \\
\hline Persistent asthma & $92(60)$ & $42(57)$ & $134(59)$ \\
Intermittent asthma & $30(19)$ & $6(8)$ & $36(16)$ \\
Seasonal asthma & $26(17)$ & $20(27)$ & $46(20)$ \\
Asthma not confirmed & $6(4)$ & $6(8)$ & $12(5)$ \\
Total & $154(100)$ & $74(100)$ & $228(100)$ \\
\hline
\end{tabular}

Table 2. Asthma Monitoring in 134 Patients With Persistent Asthma

\begin{tabular}{lccc}
\hline \hline & $\begin{array}{c}\text { Bari } \\
\text { Clinic }\end{array}$ & $\begin{array}{c}\text { Foggia } \\
\text { Clinic }\end{array}$ & Total \\
\hline Monitoring data analyzed & 65 & 19 & 84 \\
Monitoring data not analyzed & 16 & 15 & 31 \\
Monitoring procedure not applied & 11 & 8 & 19 \\
\hline
\end{tabular}

We included all new patients with poorly controlled persistent asthma, at first visit, and gave them detailed instructions on how to fill out the diary card, and adequate training on the PEF meter (Mini-Wright, Clement Clarke International, Harlow, United Kingdom). The monitoring duration was set according to the patient's asthma severity (see below).

\section{Participants}

From March 2005 to March 2006 (inclusive) at our two asthma clinics we saw 228 new adult patients with suspected asthma, referred by general practitioners (Table 1 ). Most of these patients lived in urban settings, 134 (59\%) had persistent asthma: 51 (38\%) had mild persistent asthma, $72(54 \%)$ had moderate persistent asthma, and $11(8 \%)$ had severe persistent asthma, according to the GINA 2002 guidelines. ${ }^{4}$ We applied our asthma-monitoring method with 115 (86\%) of the eligible 134 patient (Table 2). We excluded 19 patients (14\%) for various circumstantial reasons, including: staff overloading during certain visits; one patient lived permanently abroad; and one patient declined because of PEF meter cost.

We collected and analyzed the diary cards and other relevant information from 84 patients of the 115 patients monitored $(73 \%, 63 \%$ of the whole group with persistent asthma). The other 31 patients (27\% of the monitored patients, $23 \%$ of all patients with persistent asthma) were either lost to follow-up (see Table 2), for unknown reasons (29 patients), or their diary cards were not accessible at the time we analyzed the data ( 2 patients). 


\section{Retrospective Monitoring in the Management of Persistent Asthma}

\section{Diary Card}

The daily diary card (see Fig. 1) was a revised version of the example shown in the Guidelines for the Diagnosis and Management of Asthma. ${ }^{1}$ Symptom score, PEF, and medication use are recorded on this one-page, one-side, A4-size $(21 \times 30 \mathrm{~cm})$ card. The card covers 15 days. The patient writes his or her name and the year of monitoring at the top of the card, and the monitoring days (day/month) are indicated in the 15 boxes (see Fig. 1).

The scored symptoms/activities include wheezing, cough, everyday activities, and sleep. The score range is 0 to 3 for each symptom/activity, so the total symptom score range is 0 (no symptoms) to 12 (all symptoms maximally severe). Instructions on how to complete the card's symptom score section are clearly listed in a vertical box on the right side of the card, with brief examples (see Fig. 1). Patients were also asked to perform 3 successive PEF maneuvers every morning and evening, and to record the best of each set of 3 PEF values on the card. The diary card also includes a medication section and a comments section, but we did not consider that data in this analysis. The card was presented to the patient and discussed in detail at his or her first visit, in an approximately 5-10 min practice session, which usually included entering the symptom score and PEF for the visit that day.

\section{Monitoring Protocol}

Monitoring started immediately after the first visit. Monitoring duration was set according to the patient's asthma severity, and the follow-up visits were per our " 136 protocol" follow-up visit schedule for new asthma patients: first follow-up visit after one month; second visit after 3 months; third visit after 6 months. Patients with mild persistent asthma were asked to fill out the diary cards for one month; those with moderate persistent asthma for $1+3$ months; and those with severe persistent asthma for $1+3+6$ months (ie, 10 months, altogether). Patients with uncontrolled asthma symptoms (regardless of severity grade) were asked to continue to fill out the diary cards until control was achieved.

\section{Patient Survey}

A subset of 40 consecutive patients monitored at the Bari asthma clinic, between March and November 2005, were asked to anonymously answer a 3-page questionnaire on various aspects of the monitoring method, including adherence. Advance notice was given by a brief, structured telephone interview. Consent to participate in the survey was obtained from all interviewed patients. The questionnaires were mailed with a stamped addressed envelope, to return the completed questionnaire anonymously.
The questionnaire (not shown) was written in a simple and understandable style to optimize the patient response rate, and had 8 sections, on: demographics (eg, sex and age group); understanding and the filling out the diary cards, average daily time to fill out the card; the patient's opinion on the monitoring method outcomes; value for self-assessment, and the purpose and usefulness of the monitoring; the impact of the monitoring method on the patient's quality of life; nursing aspects (eg, telephone interview, appreciation of the nurse's role in asthma management); and a section for suggestions.

\section{Graphing of Symptom Score and PEF Data}

At the follow-up visits the patients returned the diary cards. In order to rapidly achieve an overall vision of the occurrence of these parameters during the period of time between successive visits and, hence, to be able to use the information gathered for clinical management and decision making, we devised and tested a method of rapidly transforming the diary-card data into an informative, easyto-read modular graph. We used graphics software (SigmaPlot 1.01, Jandel Scientific, Erkrath, Germany). In SigmaPlot we manually entered the diary-card data into a 30-day template we developed.

\section{Data Analysis}

Multiple observations are presented as arithmetic means with standard deviations. We used linear regression to analyze the linear associations between number of days of expected diary-card recording and number of days with completed entries (adherence days). We also evaluated adherence with respect to our arbitrarily selected minimum acceptable rate of completed entries: $75 \%$.

We evaluated the significance of differences in percentage (as in sex distribution) with the one-way chi-square test. When we analyzed the available data (from May 2006 to September 2006), some of the patients had not yet completed their individual asthma monitoring.

\section{Results}

One hundred fifteen adult patients with poorly controlled persistent asthma participated in the asthma monitoring protocol, based on the patient's asthma severity, as defined by the GINA 2002 guidelines. ${ }^{4}$ We analyzed the diarycard data from 84 patients (see Tables 2 and 3). Their mean age was $38.2 \pm 15.5 \mathrm{y}$, and $32(38 \%)$ were male. None of them had participated in a diary-card-based monitoring program before.

We analyzed 3 monitoring groups: mild persistent asthma (one month of monitoring) moderate persistent asthma (follow-up visits at one month and 3 months, so 120 days of 
Retrospective Monitoring in the Management of Persistent Asthma

Table 3. Asthma Monitoring Diary-Card Adherence in 84 Patients With Persistent Asthma*

\begin{tabular}{|c|c|c|c|c|c|c|c|}
\hline \multirow[b]{2}{*}{$\begin{array}{l}\text { Diary-Card Daily } \\
\text { Completion Rate }\end{array}$} & \multirow[b]{2}{*}{$\begin{array}{l}\text { Diary-Card Items } \\
\text { Completed }\end{array}$} & \multirow[b]{2}{*}{$\begin{array}{l}\text { All Patients } \\
\quad(n=84)\end{array}$} & \multicolumn{3}{|c|}{ Asthma Severity } & \multicolumn{2}{|c|}{ Sex } \\
\hline & & & $\begin{array}{c}\text { Mild } \\
(n=20) \\
(24 \%)\end{array}$ & $\begin{array}{c}\text { Moderate } \\
(n=53) \\
(63 \%)\end{array}$ & $\begin{array}{c}\text { Severe } \\
(n=11) \\
(13 \%)\end{array}$ & $\begin{array}{c}\text { Male } \\
(n=32) \\
(38 \%)\end{array}$ & $\begin{array}{c}\text { Female } \\
(n=52) \\
(62 \%)\end{array}$ \\
\hline \multirow[t]{3}{*}{$100 \%$} & PEF and symptom score & $33(39)$ & $7(35)$ & $20(38)$ & $6(55)$ & $14(44)$ & $19(37)$ \\
\hline & Symptom score only & $53(62)$ & $11(55)$ & $33(62)$ & $8(72)$ & $22(69)$ & $30(58)$ \\
\hline & PEF only & $36(43)$ & $9(45)$ & $21(40)$ & $6(55)$ & $15(47)$ & $21(40)$ \\
\hline \multirow[t]{3}{*}{$\geq 75 \%$} & PEF and symptom score & $57(68)$ & $9(45)$ & $37(70)$ & $11(100)$ & $21(66)$ & $36(69)$ \\
\hline & Symptom score only & $69(82)$ & $14(70)$ & $44(83)$ & $11(100)$ & $26(81)$ & $43(83)$ \\
\hline & PEF only & $61(73)$ & $11(55)$ & $39(74)$ & $11(100)$ & $23(72)$ & $38(73)$ \\
\hline \multirow[t]{3}{*}{$<75 \%$} & PEF and symptom score & $27(32)$ & $11(55)$ & $16(30)$ & $0(0)$ & $11(34)$ & $16(31)$ \\
\hline & Symptom score only & $15(18)$ & $6(30)$ & $9(17)$ & $0(0)$ & $6(19)$ & $9(17)$ \\
\hline & PEF only & $23(27)$ & $9(45)$ & $14(26)$ & $0(0)$ & $9(28)$ & $14(27)$ \\
\hline
\end{tabular}

* All values are no. (\%).

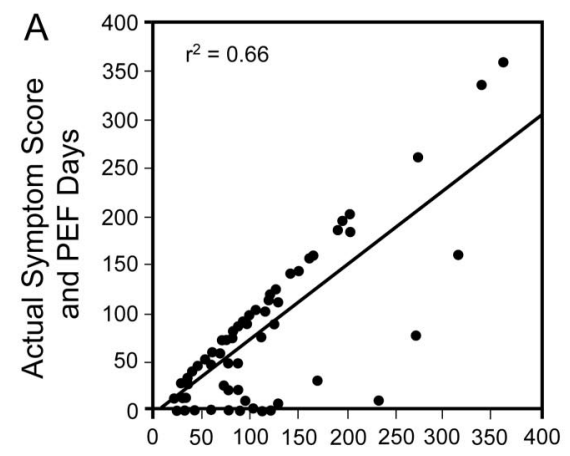

Expected Symptom Score and PEF Days

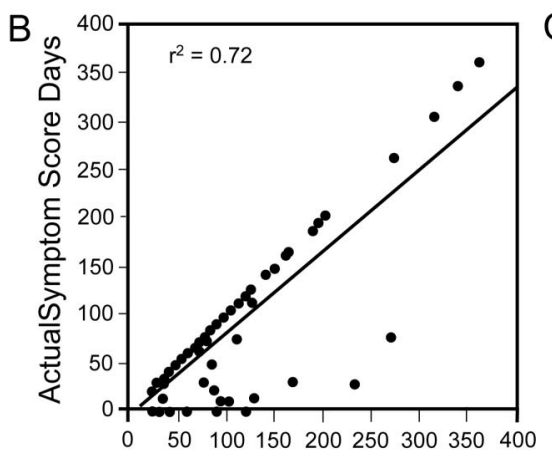

Expected Symptom Score Days

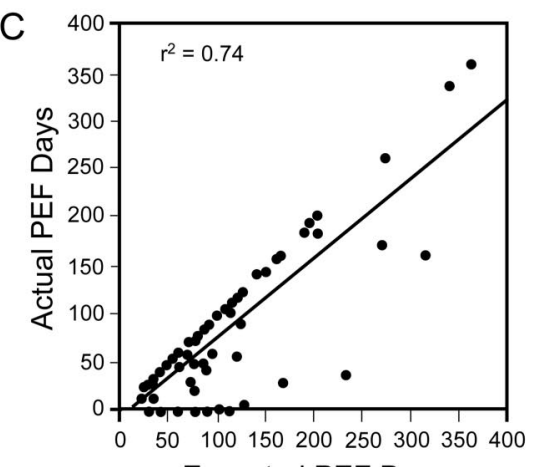

Expected PEF Days

Fig. 2. Linear regression analysis of expected versus actual days of correct diary-card completion (completed entries) for (A) both symptom score and peak expiratory flow (PEF), (B) symptom score only, and (C) PEF only.

monitoring); and severe persistent asthma (follow-up visits at one month, 3 months, and 6 months, so 300 days of monitoring). The actual number of monitoring days for any patient was seldom exactly 30,120, or 300 days, depending on the actual date of the follow-up visit.

\section{Patient Adherence}

The individual monitoring period range was 13-362 days (mean \pm SD $97.2 \pm 73.6 \mathrm{~d}$ ). The mean \pm SD number of days with completed diary-card entries (symptom score and morning and evening PEF) was $76.8 \pm 70.6$ days. Symptom score only was correctly recorded on $80.4 \pm 73.5$ days. PEF only was correctly recorded on $72.6 \pm 71.3$ days. Days with only morning or evening diary-card completion were regarded as non-adherence days.

Linear regression analysis of the relationship between days of expected diary-card completion and completed diary-card days gave a correlation coefficient $\left(\mathrm{r}^{2}\right)$ of 0.65 for symptom score plus morning and evening PEF, 0.72 for symptom score only, and 0.74 for PEF only (Fig. 2), but in the asthma-severity subgroups the correlation coefficients were 0.24 for patients with mild persistent asthma, 0.44 for patients with moderate persistent asthma, and 0.99 for patients with severe persistent asthma (Fig. 3). For symptom score only, the $\mathrm{r}^{2}$ values in the 3 groups were $0.60,0.54$, and .99 , respectively. For PEF only, the $r^{2}$ values were $0.20,0.59$, and 0.99 , respectively.

Thirty-three patients (39\%) (Table 3 and Fig. 4) had 100\% correct diary-card completion for both symptom score and PEF. Among the remaining 51 patients (61\%), 19 had $100 \%$ completion of symptom score but not PEF, and 3 had $100 \%$ completion of PEF but not symptom score.

Fifty-seven patients (68\%) had a diary-card-completion rate of $\geq 75 \%$ (our arbitrarily selected rate of acceptable completion) (see Table 3 and Fig. 4). For symptom score 

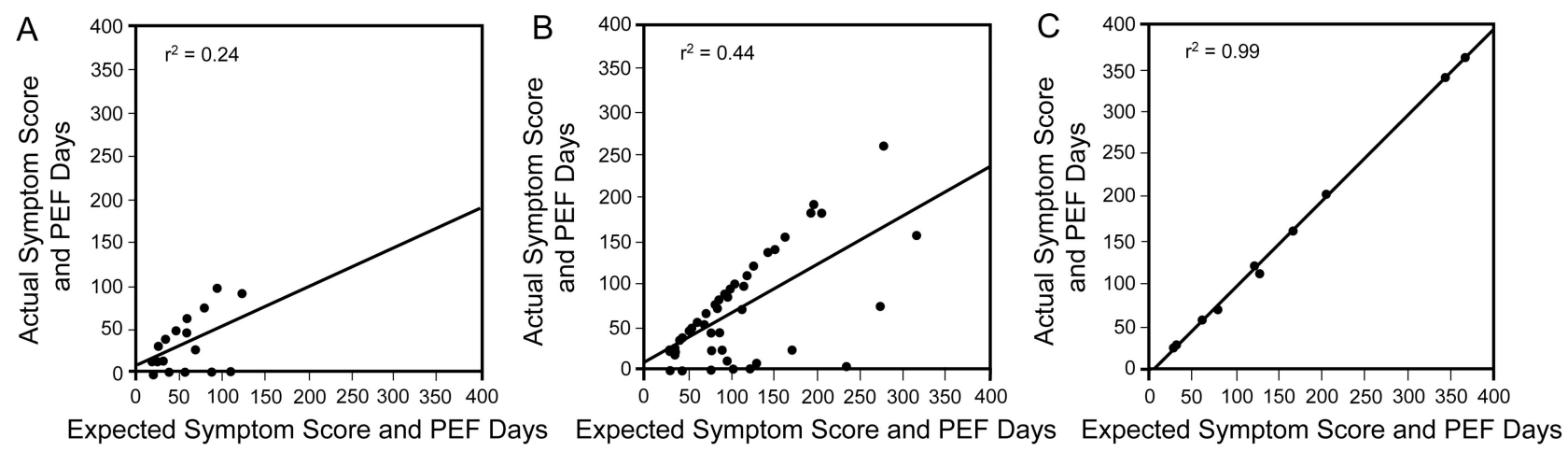

Fig. 3. Linear regression analysis of expected versus actual days of correct diary-card completion for symptom score and peak expiratory flow (PEF) in (A) patients with mild persistent asthma, (B) moderate persistent asthma, and (C) severe persistent asthma. The correlation coefficients $\left(r^{2}\right)$ increase with asthma severity.

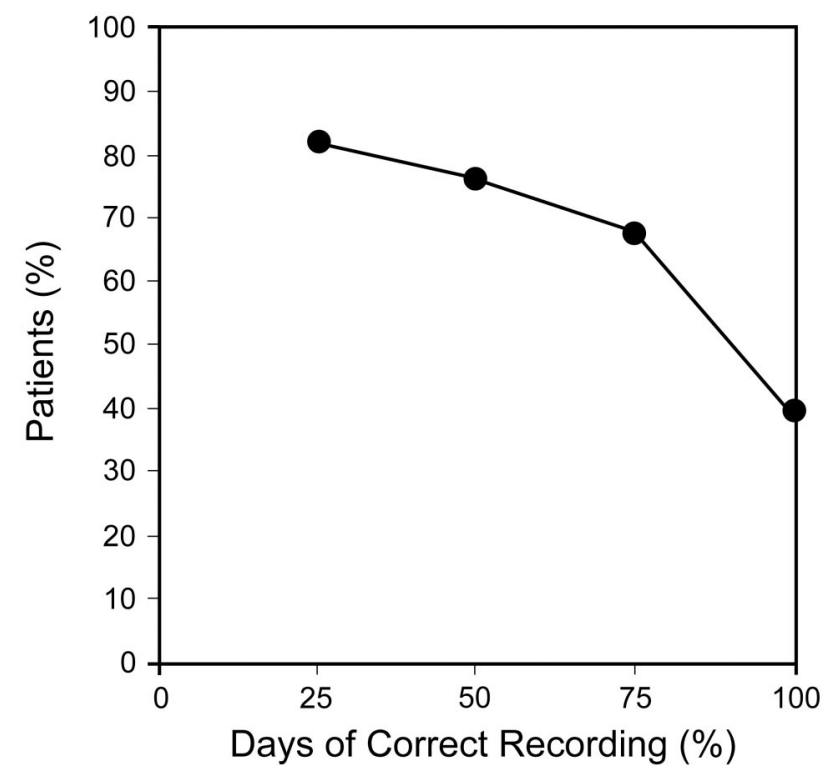

Fig. 4. Percentage of patients $(n=84)$ who achieved $25 \%, 50 \%$, $75 \%$, and $100 \%$ diary-card completion (completed entries for both symptom score and peak expiratory flow).

only, 69 patients $(82 \%)$ had a diary-card-completion rate of $\geq 75 \%$. For PEF only, 61 patients $(73 \%)$ had a diarycard-completion rate of $\geq 75 \%$. In the asthma severity groups, 9 patients $(45 \%)$ of the 20 with mild persistent asthma, 37 of the $53(70 \%)$ with moderate persistent asthma and all of the 11 patients $(100 \%)$, with severe persistent asthma had a diary-card-completion rate of $\geq 75 \%$. Notably, $6(55 \%)$ of the patients with severe asthma had $100 \%$ diary-card completion rates, while the worst performer among the other 5 patients achieved 89\%. Twentyseven patients $(32 \%)$ had diary-card-completion $<75 \%$ for symptom score or PEF or both. Of them, 23 reached the $75 \%$ threshold for symptom score but not PEF, whereas 15 reached $75 \%$ for PEF but not symptom score. Of these
27 patients, 11 had mild persistent asthma (55\% of their severity group), while 16 had moderate persistent asthma (38\% of their severity group). None of those with severe persistent asthma had diary-card-completion $<75 \%$ (see Table 3).

Interestingly, of the 29 patients who did not attend the first follow-up visit (total non-adherence), 15 had mild asthma, 14 had moderate asthma, and none had severe asthma. Only 4 patients (5\%) had no diary-card completion at all. Two patients returned diary cards with no symptom scores, and 4 patients entered no PEF values at all.

Forty-four percent of the men, and $37 \%$ of the women, had $100 \%$ diary-card completion ( $P=.67$ via chi-square). Accordingly, no statistical difference was found by the same test when the differences between the sex percentages of either those who had a $100 \%$ rate with symptom score values (but not PEF) or those who had $100 \%$ rate with PEF (but not symptom score values) were analyzed $(P=.43$ and .72 , respectively (see Table 3$)$.

\section{Patient Survey Questionnaire}

Thirty-seven patients received the questionnaire; we were unable to reach the other 3 patients. Thirty questionnaires were returned ( $81 \%$ response rate). Eleven respondents were male, 18 were female, and one did not indicate his or her sex. Three respondents $(10 \%)$ were $<20$ years old, 12 (40\%) were between 20 and 40 years old, 13 (43\%) were between 41 and 60 years old, and $2(7 \%)$ were $>60$ years old. Table 4 shows the responses we analyzed in this study. In particular, we assessed that the daily time required to fill out the diary card was as low as $3 \mathrm{~min} 30 \mathrm{~s} \pm 2 \mathrm{~min}$ $30 \mathrm{~s}$ on average. Three patients provided a non-numerical answer to this question (eg, very little), and one patient gave no answer. 


\section{Retrospective Monitoring in the Management of Persistent Asthma}

Table 4. Patient Survey Results*

\begin{tabular}{|c|c|c|c|}
\hline & $\begin{array}{l}\text { Yes } \\
\text { no. }(\%)\end{array}$ & $\begin{array}{l}\text { No } \\
\text { no. }(\%)\end{array}$ & $\begin{array}{c}\text { No } \\
\text { Answer } \\
\text { no. }(\%)\end{array}$ \\
\hline \multicolumn{4}{|l|}{ 1. The monitoring cards } \\
\hline - Do you think they are understandable? & $29(97)$ & $1(3)$ & 0 \\
\hline - Do you think they are complete? & $25(83)$ & $4(13)$ & $1(3)$ \\
\hline - Do you think they are useful? & $30(100)$ & 0 & 0 \\
\hline - Do you think they give you enough information on how to fill them out well? & $24(80)$ & $4(13)$ & $2(7)$ \\
\hline - Do you think they are difficult to fill out? & $3(10)$ & $26(87)$ & $1(3)$ \\
\hline \multicolumn{4}{|l|}{$\begin{array}{l}\text { 2. How many minutes, per day, does it take to fill out the cards? [mean } \pm \text { SD } 3 \\
\min 30 \mathrm{~s} \pm 2 \min 30 \mathrm{~s} \text { ] }\end{array}$} \\
\hline \multicolumn{4}{|l|}{ 3. The results from the cards } \\
\hline $\begin{array}{l}\text { - Do you think it is important to know the symptom score and PEF values } \\
\text { during therapy? }\end{array}$ & $30(100)$ & 0 & 0 \\
\hline $\begin{array}{l}\text { - The values on the monitoring cards are transformed into graphs. Do you think } \\
\text { it may be useful to you to get these graphs? }\end{array}$ & $23(77)$ & $7(23)$ & 0 \\
\hline - Do you think they are useless to you and useful only to the medical staff? & $12(40)$ & $18(60)$ & 0 \\
\hline \multicolumn{4}{|l|}{ 4. The cards serve for: } \\
\hline - Educating asthma self-management? & $19(63)$ & $8(27)$ & $3(10)$ \\
\hline - Doing research and statistical studies? & $28(93)$ & $1(3)$ & $1(3)$ \\
\hline - Allowing the medical personnel to take better care of you? & $29(97)$ & 0 & $1(3)$ \\
\hline - Monitoring your conditions between control visits? & $29(97)$ & $1(3)$ & 0 \\
\hline - Predicting exacerbations? & $22(73)$ & $6(20)$ & $2(7)$ \\
\hline - Improving the patient/doctor relationship? & $21(60)$ & $7(23)$ & $2(7)$ \\
\hline - They are of little use. & $3(10)$ & $24(80)$ & $3(10)$ \\
\hline - They are of no use. & 0 & $26(87)$ & $4(13)$ \\
\hline \multicolumn{4}{|l|}{ 5. Since you have been using the cards: } \\
\hline - Do you find it easier to remember taking all your medications during the day? & $22(73)$ & $8(27)$ & 0 \\
\hline - Do you feel more at ease knowing that in this way you can monitor yourself? & $23(73)$ & $4(13)$ & $3(10)$ \\
\hline - Do you feel more comfortable and relaxed? & $18(60)$ & $8(27)$ & $4(13)$ \\
\hline - Do you think it is worth doing it (considering the time needed, etc)? & $24(80)$ & $1(3)$ & $5(17)$ \\
\hline \multicolumn{4}{|l|}{ 6. Other issues } \\
\hline a. According to you, were the explanations received by phone clear enough? & $11(37)$ & $18(60)$ & $1(3)$ \\
\hline b. Do you think that this questionnaire could have been filled out also by phone? & $11(37)$ & $19(63)$ & 0 \\
\hline \multicolumn{4}{|l|}{ c. According to you, why have you been asked to fill out this questionnaire? $\dagger$} \\
\hline - To get an overall judgment about our work. & $24(80)$ & 0 & 0 \\
\hline - To involve you and improve compliance and cooperation. & $16(53)$ & 0 & 0 \\
\hline - To carry out statistical studies. & $19(63)$ & 0 & 0 \\
\hline - I do not know. & $1(3)$ & 0 & 0 \\
\hline - Other & $3(10)$ & 0 & 0 \\
\hline
\end{tabular}

${ }_{* n} n=30$ patients. This table reflects the language used in the questionnaire, which was deliberately simple. The questionnaire also included a nursing section and other information, omitted here $\dagger$ Multiple answers possible.

\section{Data Graphing}

At the follow-up visits the patients returned their filled out diary cards, and we entered the symptom score and PEF data and processed and graphed them immediately. Figure 5 depicts a graph from 2 diary cards, covering one month of monitoring.

In SigmaPlot we developed a 30-day template, which could be rapidly modified and the diary-card data entered in less than 5 minutes, for a one-month graph. The time required was slightly longer for several months of data.
The training time to achieve rapid graphing skill was approximately 2 hours for an average computer user.

In Figure 5, the $\mathrm{X}$ axis shows 30 sequential days of monitoring; we decided not to use the actual monitoring dates, to simplify data entry and improve graph readability. The monitoring start and end dates, and the patient's name, are shown in a heading above the graph (not shown in Figure 5). The left $\mathrm{Y}$ axis shows the PEF scale. Since PEF ranges widely according to sex, height, age, and clinical conditions, ${ }^{12}$ the left Y-axis range $(200-700 \mathrm{~L} / \mathrm{min})$ is adjusted in relation to the individual patient data being 


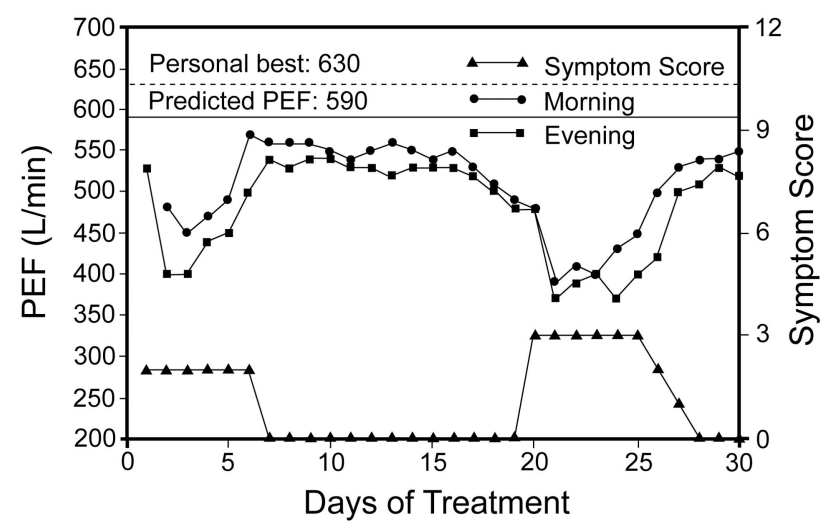

Fig. 5. Our format for graphing asthma monitoring data (peak expiratory flow [PEF] and symptom score). This graph is of data from a patient with moderate persistent asthma. There are asthma exacerbations (PEF dropped and symptom score rose) around days 1-7 and 19-28. Note that, as sometimes happens, evening PEF appears to be consistently lower than morning PEF. (Morning PEF should be consistently lower than evening PEF, but sometimes it is the other way around. This and other anomalies can be easily detected by this monitoring method.) The horizontal dotted indicates the patient's personal best PEF. The horizontal solid indicates the patient's predicted PEF.

processed, to improve graph readability and facilitate rapid visual pattern recognition. ${ }^{11}$ The mean actual left Y-axis lower and upper limits were $208.9 \pm 38.5 \mathrm{~L} / \mathrm{min}$ and $640 \pm 83.7 \mathrm{~L} / \mathrm{min}$, respectively (as calculated from the data from 63 of the 84 patients). The graph also shows the predicted PEF value (according to Nunn and Gregg ${ }^{13}$ ) and the patient's personal best PEF, with both horizontal lines (the personal-best line is dashed, and the predicted PEF is solid) and text. The right $\mathrm{Y}$ axis shows the symptom score scale (range 0-12) (see Fig. 5). To graph monitoring periods longer than 30 days we use multiple 30 -day graphs.

\section{Discussion}

Home asthma monitoring with daily diary-card recording of symptom score and/or pulmonary-function variables (generally PEF) has been in use for 30 years $^{14}$ and has been incorporated into some of the most influential guidelines on asthma management. ${ }^{1-4}$ However, there is still considerable disagreement on several aspects of asthma monitoring, from overall usefulness to standardization of graphic data rendering.7,11,15-17 Asthma home monitoring has been applied in 4 settings: self-management, in association with an asthma action plan $^{5-7}$; management of asthma patients by clinicians, including diagnosis refining, and retrospective therapy assessment ${ }^{8-11}$; clinical trials and clinical research, where it is one of the most widely used investigation tools and a valuable source of information ${ }^{18-22}$; and occupational asthma. ${ }^{23-24}$
Our study dealt with retrospective monitoring of patients with poorly controlled persistent asthma and no previous experience of home monitoring, in a clinical management setting. We use the word "retrospective" to mean the analysis of recorded recent events, to evaluate the outcomes of management measures, to affect future diseasemanagement recommendations. In particular, we analyzed the patients' adherence to the method adopted (also by means of a questionnaire, answered anonymously). Moreover, we developed a user-friendly computer-based technique to quickly graph clinically relevant data from the diary cards and facilitate rapid visual pattern recognition.

As for the number of patients whose recording cards were analyzed (84), some restrictions may indeed arise from this relatively limited sample size. However, we believe that this problem is of minor importance and does not undermine the internal validity of the study, since the differences between groups (particularly between the asthmaseverity groups) appear both too large and too consistent to be attributed to chance.

Moreover, the external validity of our study may be partly limited by possible selection biases, particularly asthma severity, which may increase adherence, as our findings suggest (see below). In fact, it is reasonable to assume that our local general practitioners refer their most problematic patients to our university clinics, since 83 (62\%) of the 134 patients eligible for our asthma-monitoring method had moderate or severe asthma. Other possible biases include the patients' perception of their asthma and their socioeconomic status, which probably influence adherence, but these issues did not fall within the purposes or the potential of our study and would require further investigation and a different study design. However, again, we think that these are relatively minor limitations and do not invalidate our results.

\section{Adherence}

Several studies have addressed patient adherence, but have produced an overall inconclusive and sometimes conflicting picture. Thus, while, on one hand, symptom score and PEF monitoring are regarded as essential not only in clinical trials but also in ordinary asthma management programs, 7,17 on the other hand, the method itself is often thought to be inherently faulty due to poor adherence, if not falsification. This has sometimes caused a kind of "nihilistic" attitude toward home monitoring, based on the perception that satisfactory adherence cannot be achieved. ${ }^{25}$ One study, in which (as in several others) patients were unaware they were using devices that recorded the measurements, concluded that the quality of data completion is often poor, ${ }^{26}$ although there was only a $2 \%$ discrepancy between the patients' handwritten reports and the electronic record in the PEF morning measurements, and 9\% 


\section{Retrospective Monitoring in the Management of Persistent Asthma}

in the evening measurements. ${ }^{26}$ Another investigation, in which patients were asked to measure PEF, forced vital capacity, and $\mathrm{FEV}_{1}$ with hand-held spirometer (again, with data storage unknown to them), and to record the values on conventional diary cards, had $70 \%$ completed entries, of which $4 \%$ were fabricated. Those seem to us acceptable rates, given the complexity of the measurements performed, whereas Chowienczyk et al concluded that conventional asthma diary cards contain a high number of fabricated or retrospective entries. ${ }^{27} \mathrm{~A}$ third study, in 20 patients with steady-state asthma, found that only $54 \%$ of the expected PEF values were recorded by the patient, and adherence declined over the 3-month study period. Moreover, a substantial $22 \%$ of the entered values were fabricated. ${ }^{28} \mathrm{How}-$ ever, the fact that the patients were clinically stable might have reduced their motivation to adhere to a method that, as in our setting, may have been perceived by patients with poorly controlled asthma as instrumental in gaining clinical improvement.

In contrast, another study, with a user-friendly electronic PEF monitor and twice daily PEF measurements, by patients aware that the data were stored, reported an overall $89 \%$ adherence over 72 weeks. ${ }^{29}$ However, the 61 patients in that study had poorly controlled asthma, and their expectations about monitoring were probably high. Reddel et al concluded that the context in which monitoring is undertaken may influence adherence and that the patients' perception of usefulness (and hence better adherence) may have been enhanced by visual presentation and discussion of graphed data at control visits every 8 weeks. ${ }^{29}$

We believe that, in our strictly clinically oriented setting, we had an acceptable adherence rate: our overall correlation coefficient between expected and actual days of diary-card completion (completed entries) was 0.65 . Moreover, 33 (39\%) of the 84 patients had $100 \%$ symptom score and PEF diary-card completion, and $68 \%$ had $\geq 75 \%$ completion, which might be regarded as poor in research but is reasonably good in a clinical management setting. Furthermore, adherence was strikingly correlated with asthma severity, being rather poor in those with mild asthma, higher in those with moderate asthma, and impressively good in those with severe asthma. All 11 patients with severe asthma had $\geq 75 \%$ completion, and 6 of them had $100 \%$ complete entries. This is particularly noteworthy because, in our follow-up and monitoring "136 protocol" patients with severe asthma perform a much longer monitoring (300 d vs 120 days in patients with moderate asthma, and only 30 days in patients with mild asthma). Therefore, our results are in agreement with studies in which poorly controlled but highly motivated patients carried out home symptom and PEF monitoring for prolonged periods with good adherence, which declined only marginally over time. ${ }^{25,29}$ However, our results are in striking disagreement with other studies of stable chronic asthma patients or with expert trialists, which indicated that adherence was generally poor and worsened during prolonged monitoring, and that falsification was rampant. ${ }^{28,30}$ Also, we found that adherence was consistently higher for symptom score than for PEF (see Table 3): $82 \%$ of the patients had $\geq 75 \%$ adherence with symptom score, but only $73 \%$ with PEF.

\section{Patient Survey}

Our 30 survey responses offered an interesting insight into our patients' point of view on our asthma monitoring method. They found the diary card useful, comprehensible, and exhaustive. However, about $10 \%$ of them found it either difficult to fill out or that the information provided was inadequate for correct data entering, which indicates an opportunity for further improvement of this important asthma management tool. The daily required time to fill out the diary card was remarkably short: less than 4 min on average (see Table 4), which suggests that a larger investment of time may not be a major cause of poor adherence in home monitoring, in motivated patients.

Moreover, the vast majority of the respondents thought that it was important to have access to the graphs generated at the follow-up visits, which are in fact regularly shown to and discussed with the patients; this confirms that the patients' perceived usefulness of monitoring is enhanced by presentation and discussion of graphed data at each follow-up visit. ${ }^{25}$ Receiving a hard copy of the graph appeared to be less important (see Table 4). Regarding the reasons the monitoring method was considered useful, the great majority of the patients believed that it enabled the clinicians to give them better treatment, by precisely documenting their conditions between consecutive visits. But, whereas $92 \%$ of them thought that the method could improve the quality of the patient/doctor relationship, only $60-70 \%$ knew that monitoring could also help asthma self-management and exacerbation prevention, perhaps consistently reflecting our approach in using the card diary-card method essentially for retrospective assessment. That also highlights the possibility that self-management is an aspect of asthma management that might need improvement at our clinics.

Most of the respondents also thought that the monitoring method was aimed at research or statistical purposes, which is a misperception, probably reflecting the fact that prolonged home monitoring is not very common, at least in Italy. Moreover, most patients found the monitoring method emotionally reassuring, because they could better "control themselves," hence confirming a basically positive disposition toward self-management, which needs to be exploited. Also, ease in coping with their often complex medication schedule was another advantage perceived by most patients. 


\section{Retrospective Monitoring in the Management of Persistent Asthma}

\section{Graphing and Visual Pattern Recognition}

Effective and synthetic graphing is essential for the complete understanding and utilization of the copious information that asthma monitoring can disclose. In their thorough review of the subject, Reddel et al rightly pointed out that the important "bottleneck" in the graphing is how to combine clarity and plasticity, usually achieved via computerized treatment of the data, with ease and rapidity in generating the graphs, which they instead associate with manual entry. ${ }^{11}$ We used a robust yet flexible scientific graphics platform (SigmaPlot) that allowed rapid data entry ( $\leq 5 \mathrm{~min}$ for 30 days of monitoring data) in a computerized form at follow-up visits, and high quality, immediate graphing of morning and evening PEF and symptoms on one graph that effectively shows the patient's status over one month (see Fig. 5). This method, which we have used for many years, appears to be reliable and simple enough to be clinically useful. Moreover, the data can be statistically analyzed within SigmaPlot versions with improved statistical power (up to version 11) or in other kinds of software, allowing further analysis for research.

Lack of standardization in graph dimensions and other features important for development of visual pattern recognition skills has been another major problem. Particularly, horizontal compression of the graph impressively enhances detection of exacerbations. ${ }^{11}$ We adopted a 30days format with the classic 1/1.5 height/width ratio, which is lower than that of the Woolcock Institute of Medical Research format $(1 / 0.82)^{11}$ and the occupational asthma format (1/0.39). ${ }^{11,24}$ Our graphing system, however, offers the possibility of selecting a PEF scale range on the left $\mathrm{Y}$ axis appropriate for the patient, easily attainable in SigmaPlot, compensating for the lower compression degree, and providing unequivocal detection of exacerbations (see Fig. 5). Moreover, simultaneous display of both morning and evening PEF and symptom score maximizes the amount of displayed asthma-monitoring information and facilitates visual appreciation of diurnal PEF variability and identification of anomalies, as in Figure 5. And the inclusion of both predicted PEF and personal best PEF allows immediate interpretation of the PEF curves.

Human visual pattern recognition processes are highly sophisticated and reliable, so PEF and symptom score graph standardization and optimization of the readability, clarity, and type and amount of information in the graph stimulate the development of visual pattern recognition skills in clinicians. This is likely to increase clinicians' interest in the monitoring data and their ability to use the data in decision making, with probable improvement in the care provided. That, in turn, is likely to be positively perceived by patients and to reinforce their adherence to the monitoring method and other aspects of management, ${ }^{11}$ as our data suggest (see Table 4).

\section{Conclusions}

Asthma monitoring of symptom score and PEF, even when carried out with an inexpensive diary-card device, is useful and feasible in clinical practice, and patient adherence is acceptably high. Thus, our results seem to contradict the conclusions of at least some previous studies, which asserted that diary-card completion of symptom score and PEF, although desirable as an asthma management tool, is virtually impossible, due to poor patient adherence, particularly over a prolonged period.

In particular, our study focused on retrospective monitoring and immediate data analysis with relevance to decision making at follow-up visits. Indeed, our results suggest that, in this context, symptom score and PEF monitoring is a valuable management tool in patients with poorly controlled persistent asthma, in patients with moderate asthma and particularly severe asthma, in whom we found very high adherence over prolonged periods. We would place a lower priority on retrospective monitoring in patients with mild persistent asthma, because our results suggest poor adherence in those patients and an arguably unfavorable cost/benefit ratio. Moreover, monitoring should be carried out within a definite follow-up protocol in order to contribute to asthma management. We propose approximately 4 months for patients with moderate persistent asthma and 10-12 months for patients with severe asthma.

However, monitoring may be of great usefulness in all asthma patients, including patients with mild persistent asthma, patients with intermittent asthma, and patients with seasonal asthma, as a prospective self-management tool aimed at preventing exacerbations, possibly in association with an asthma action plan. We also believe that substantial efforts are warranted to increase the awareness of the importance of symptom score and PEF monitoring in asthma management at the primary care level, as opposed to the view that monitoring occurs only at specialized clinics or in clinical trials.

Improving the graphing of asthma monitoring data appears to be very important in exploiting the clinical potential of this information. Finally, standardization of symptom score and PEF graphs should be considered a priority, if monitoring is to be used more effectively in the management of moderate and severe persistent asthma, and to develop clinicians' pattern recognition skills.

\section{REFERENCES}

1. US National Institutes of Health; National Hearth, Lung, and Blood Institute; National Asthma Education and Prevention Program. Expert Panel Report 2. Guidelines for the diagnosis and management of 


\section{Retrospective Monitoring in the Management of Persistent Asthma}

asthma. NIH Publication 97-4051. Bethesda: National Institutes of Health; 1997.

2. Global Initiative for Asthma (GINA). Global strategy for asthma management and prevention. http://www.ginasthma.com/guideline item.asp? $11=2 \& 12=1 \&$ intid=60. Accessed March 9, 2011

3. Scottish Intercollegiate Guidelines Network. 101 - British guideline on the management of asthma: a national clinical guideline. Revised June 2009. http://www.sign.ac.uk/pdf/sign101.pdf. Accessed March 9, 2011.

4. Global Initiative for Asthma (GINA). 2002 original: workshop report, global strategy for asthma management and prevention. http:// www.ginasthma.com/guidelineitem.asp? $11=2 \& 12=1 \&$ intid $=82$. Accessed March 9, 2011.

5. D'Souza W, Crane J, Burgess C, Te Karu H, Fox C, Harper M, et al. Community-based asthma care: trial of a "credit card" asthma selfmanagement plan. Eur Respir J 1994;7(7):1260-1265.

6. Ignacio-Garcia JM, Gonzalez-Santos P. Asthma self-management education program by home monitoring of peak expiratory flow. Am J Respir Crit Care Med 1995;151(2 Pt 1):353-359.

7. Gibson PG, Powell H, Wilson A, Abramson MJ, Haywood P, Bauman A, et al. Self-management education and regular practitioner review for adults with asthma. Cochrane Database Syst Rev 2002; (3):CD001117.

8. Mitchell DM, Gildeh P, Dimond AH, Collins JV. Value of serial peak expiratory flow measurements in assessing treatment response in chronic airflow limitation. Thorax 1986;41(8):606-610.

9. Johnston SL, Pattemore PK, Sanderson G, Smith S, Lampe F, Josephs L, et al. Community study of role of viral infections in exacerbations of asthma in 9-11 year old children. BMJ 1995;310(6989): 1225-1229.

10. Reddel H, Ware S, Marks G, Salome C, Jenkins C, Woolcock A. Differences between asthma exacerbations and poor asthma control. Lancet 1999;353(9150):364-369.

11. Reddel HK, Vincent SD, Civitico J. The need for standardization of peak flow charts. Thorax 2005;60(2):164-167.

12. Quanjer PH, Lebowitz MD, Gregg I, Miller MR, Pedersen OF. Peak expiratory flow: conclusions and recommendations of a working party of the European Respiratory Society. Eur Respir J 1997;10(Suppl 24):2S-8S

13. Nunn AJ, Gregg I. New regression equations for predicting peak expiratory flow in adults. BMJ 1989;298(6680):1068-1070.

14. Prior JG, Cochrane GM. Home-monitoring of peak expiratory flow rate using mini-Wright peak flow meter in diagnosis of asthma. J R Soc Med 1980;73(10):731-733.

15. Brand PLP, Roorda RJ. Usefulness of monitoring lung function in asthma. Arch Dis Child 2003;88(11):1021-1025.

16. Brusasco V. Usefulness of peak expiratory flow measurements: is it just a matter of instrument accuracy? Thorax 2003;58(5):375-376.
17. Reddel HK. Peak flow monitoring in clinical practice and clinical asthma trials. Curr Opin Pulm Med 2006;12(1):75-81.

18. Spector SL, Smith LJ, Glass M; ACCOLATE Asthma Trialists Group. Effects of 6 weeks of therapy with oral doses of ICI 204,219, a leukotriene $\mathrm{D}_{4}$ receptor antagonist, in subjects with bronchial asthma. Am J Respir Crit Care Med 1994;150(3):618-623.

19. Bellia V, Cuttitta G, Cibella F, Vignola AM, Crescimanno G, D'Accardi $\mathrm{P}$, et al. Effect of ageing on peak expiratory flow variability and nocturnal exacerbations in bronchial asthma. Eur Respir J 1997;10(8):1803-1808.

20. Boushey HA, Sorkness CA, King TS, Sullivan SD, Fahy JV, Lazarus $\mathrm{SC}$, et al. Daily versus as-needed corticosteroids for mild persistent asthma. N Engl J Med 2005;352(15):1519-1528.

21. Frey U, Brodbeck T, Majumdar A, Taylor DR, Town GI, Silverman M, Suki B. Risk of severe asthma episodes predicted from fluctuation analysis of airway function. Nature 2005;438(7068):667-670.

22. O'Byrne PM, Bisgaard H, Godard PP, Pistolesi M, Palmqvist M, $\mathrm{Zhu} \mathrm{Y}$, et al. Budesonide/formoterol combination therapy as both maintenance and reliever medication in asthma. Am J Respir Crit Care Med 2005;171(2):129-136.

23. Moscato G, Godnic-Cvar J, Maestrelli P, Malo J-L, Burge PS, Coifman R. Statement on self-monitoring of peak expiratory flows in the investigation of occupational asthma. Eur Respir J 1995;8(9):16051610 .

24. Bright P, Burge PS. Occupational lung disease. 8: The diagnosis of occupational asthma from serial measurements of lung function at and away from work. Thorax 1996;51(8):857-863.

25. Reddel HK, Toelle BG, Marks GB, Ware SI, Jenkins CR, Woolcock AJ. Analysis of adherence to peak flow monitoring when recording of data is electronic. BMJ 2002;324(7330):146-147.

26. Hyland ME, Kenyon CAP, Allen R, Howarth P. Diary keeping in asthma: comparison of written and electronic methods. BMJ 1993; 306(6876):487-489.

27. Chowienczyk PJ, Parkin DH, Lawson CP, Cochrane GM. Do asthmatic patients correctly record home spirometry measurements? BMJ 1994;309(6969):1618.

28. Verschelden P, Cartier A, L'Archevêque J, Trudeau C, Malo J-L. Compliance with and accuracy of daily self-assessment of peak expiratory flows (PEF) in asthmatic subjects over a three month period. Eur Respir J 1996;9(5):880-885.

29. Reddel HK, Jenkins CR, Marks GB, Ware SI, Xuan W, Salome CM, et al. Optimal asthma control, starting with high doses of inhaled budesonide. Eur Respir J 2000;16(2):226-235.

30. Côté J, Cartier A, Malo J-L, Rouleau M, Boulet L-P. Compliance with peak expiratory flow monitoring in home management of asthma. Chest 1998;113(4):968-972. 